\title{
SIMULACIÓN DE MANEJO SILVÍCOLA EN BASE A UMBRALES DE COBERTURA EN SISTEMAS SILVOPASTORILES COMPUESTOS POR Festuca pallescens y Pinus ponderosa.
}

Federico Letourneau ${ }^{1}$, Gonzalo Caballé ${ }^{1}$, Ernesto Andenmatten ${ }^{2}$, Nicolás De Agostini ${ }^{3}$.

\section{RESUMEN}

Los sistemas silvopastoriles están sujetos a las interacciones que se generan entre sus componentes. Para asegurar el éxito productivo de estos sistemas el balance neto de las interacciones árbol-pasto debe ser neutro o positivo, de prevalecer la competencia el sistema no es sustentable en el tiempo.

Festuca pallescens, una de las principales especies forrajeras de la Patagonia Argentina, mostró buena adaptación a crecer bajo dosel de pino ponderosa (Pinus ponderosa) en sistemas silvopastoriles. Estudios ecofisiológicos indican que el umbral crítico de cobertura arbórea para el cual el balance neto de las interacciones es neutro o positivo se encuentra próximo al $70 \%$. Por sobre este nivel, la fuerte competencia por radiación se contrapone con la facilitación generada por la disminución de la demanda atmosférica.

Las prácticas silvícolas de raleo permiten regular los niveles de cobertura para no sobrepasar el umbral crítico. Por lo tanto, se requiere de indicadores de fácil determinación para poder aplicar estas prácticas en el momento adecuado.

Es imprescindible conocer como cambia la cobertura a medida que los árboles se desarrollan. Esto se puede lograr mediante el empleo de modelos de crecimiento y rendimiento forestal. El simulador Piltriquitron, desarrollado por INTA, para pino ponderosa, si bien predice el crecimiento y el rendimiento, hasta el momento no simulaba los niveles de cobertura arbórea asociados.

Buscando completar este faltante de información se estableció la relación que existe entre parámetros estructurales del rodal y la cobertura en 61 rodales de pino ponderosa distribuidos entre los $36,5^{\circ}$ y $42,5^{\circ}$ LS. Luego se incorporó esta relación al simulador, y considerando los umbrales críticos se simularon esquemas de raleos para mantener los niveles de cobertura adecuados.

El mejor ajuste teniendo en cuenta el coeficiente de correlación, la distribución de

\footnotetext{
1 INTA EEA Bariloche, CC277 (8400) Bariloche, Río Negro, Argentina. fletourneau@bariloche.inta.gov.ar y gcaballe@bariloche.inta.gov.ar

2 INTA Centro regional Patagonia Norte, Argentina. eandenmatten@correo.inta.gov.ar

3 Servicio de Prevención y Lucha de Incendios Forestales, Pcia Río Negro, Argentina: ndeagostini@gmail.com
} 
los errores de una muestra de validación y algunos parámetros prácticos relacionados con la aplicación al simulador se encontró entre apertura del dosel e índice de densidad relativa de Curtis (1982). Las simulaciones realizadas muestran que iniciando el ciclo forestal con 1.111 árboles ha-1 ${ }^{-1}$ para no sobrepasar el umbral crítico es necesario aplicar dos raleos, llegando al turno ( $45 \mathrm{~cm}$ de diámetro medio cuadrático) con 75 árboles ha-1 en un sitio de calidad intermedia, con una producción bruta total de $280 \mathrm{~m}^{3} \mathrm{ha}^{-1}, \mathrm{y} 190 \mathrm{~m}^{3} \mathrm{ha}^{-1}$ de volumen comercial incluyendo raleos. Los resultados aquí obtenidos contrastan con

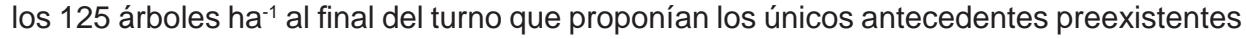
para Patagonia. Estos resultados muestran que para sostener el silvopastoreo sería necesario resignar fuertemente la producción de madera por unidad de superficie.

Palabras clave: Festuca pallescens, Pinus ponderosa, Manejo Silvopastoral 


\section{SUMMARY}

Silvopastoral systems are subject to interactions generated between its components. The productivity of these systems is dependent on the interaction of environmental and management factors that affect the understory sward. If competition prevails the systems becomes unsustainable.

Festuca pallescens, a main forage species in Patagonia Argentina, showed good adaptation to grow under canopy of ponderosa pine (Pinus ponderosa) in silvopastoral systems. Ecophysiological studies indicate that the critical threshold of tree canopy cover for which the net balance between positive and negative interactions are cero or positive, is close to $70 \%$. Above this level, the facilitation effect generated by the lower atmospheric demand under the trees is lost due to a strong competition for radiation.

Silvicultural management can regulate the levels of canopy cover to not exceed the critical threshold. Therefore, it requires easy indicators to measure in order to apply these practices at the right time. At the same time, it is essential to know how coverage changes as trees grow. This can be achieved through the use of forest growth and yield models. Piltriquitron, is the model developed by INTA for ponderosa pine to predicts the growth and yield, but did not predict, until now, the levels of tree cover associated.

To complete this missing information we established the relationship between structural parameters of the stand and coverage in 63 ponderosa pine stands distributed between $36,5^{\circ} \mathrm{SL}$ and $42,5^{\circ} \mathrm{SL}$. After that, we joined this relationship to the Piltriquitron model, and considering the critical threshold schemes were simulated thinning to maintain adequate levels of coverage.

The best fit taking into account the correlation coefficient, the distribution of errors in a validation sample and some practical parameters related to the application to the model was found between coverage and Curtis relative density index. Simulations showed that the forestry cycle starting with 1,111 trees $^{-1}$, not to exceed the critical threshold is necessary to apply two thinnings, reaching the turn (mean diameter $45 \mathrm{~cm}$ square) with 75 trees ha-1 on a site of intermediate quality, with a total production of $280 \mathrm{~m}^{3} \mathrm{ha}^{-1}$, and $190 \mathrm{~m}^{3} \mathrm{ha}^{-1}$ of commercial volume, including thinning. The results obtained here contrast with the 125 trees $^{-1} \mathrm{a}^{-1}$ at the end of the shift that proposed the only existing record for Patagonia. These results show that it is necessary to sustain the silvopastoral strongly resign wood production per unit area.

Key words: Festuca pallescens, Pinus ponderosa, Silvopastoral management 


\section{INTRODUCCIÓN}

En una vasta región de la Norpatagonia Andina Argentina la ganadería ha sido y continúa siendo un modo tradicional y económicamente rentable del uso de la tierra. En muchos campos, la gramínea cespitosa Festuca pallescens es el principal recurso forrajero y el más extendido geográficamente.

En estos campos también se ha plantado Pinus ponderosa, en sitios relativamente marginales para la actividad ganadera, pero a veces se la ha visto como actividad que puede competir en el uso de la tierra. Esto se debe a que las condiciones del sotobosque en las plataciones con fines industriales, con un intenso sombreado del suelo, no permite sostener el crecimiento de los pastos. Sin embargo con un adecuado manejo de la cobertura arbórea es posible compatibilizar ambas producciones, hacer un uso más eficiente del espacio y mejorar la economía rural.

Los sistemas silvopastoriles combinan estas actividades que pueden resultar en mejoras de productividad de la actividad ganadera a corto plazo, por el incremento del bienestar animal, como el que podría lograrse al disminuir la temperatura interna del animal en días de calor (Valtorta et al., 1997), o al incrementar su sensación térmica por reducción de la velocidad del viento (Brandle et al., 2001). A largo plazo se diversifica la producción de las actividades rurales y se puede obtener dividendos por venta de la madera. En los sistemas silvopastoriles los árboles y pastos ejercen influencia uno sobre otro, con consecuencias sobre su desempeño.

El estudio de la interacción entre plantas reconoce la existencia de un balance entre facilitación y competencia, donde ambos efectos son concurrentes, aunque uno de estos prevalece sobre el otro (Holmgren et al., 1997). Estas interacciones han sido estudiadas también entre árboles y pastos, donde el componente arbóreo puede tener un efecto positivo sobre los pastos al mejorar su condición hídrica (Mosquera -Losada et al., 2004), aunque la radiación sea menor que a cielo abierto. Sin embargo a niveles de sombreado intenso este beneficio puede no compensar la reducción de luz y las matas de F. pallescens pueden sufrir una caída notable de su productividad. Estos efectos han sido estudiados por Fernández (2003) y Fernández et al. (2006) encontrando que $P$. ponderosa ejerce un efecto positivo sobre esta especie, por que mejora su estado hídrico a la sombra en épocas de alta demanda estival. Aunque también midieron disminución de crecimiento en matas de F. pallescens cuando se supera el $70 \%$ de cobertura.

Si bien se dispone de esta información que permite realizar recomendaciones en función de la cobertura actual de los rodales, son escasas las propuestas de esquemas de raleo para Sistemas Silvopastoriles en la zona de estudio. Laclau (1999) analizó la viabilidad económica de estos sistemas, y utilizó rangos de densidad relativa, como guía para el manejo del componente herbáceos, pero menciona que desconoce el efecto que tiene este rango de condiciones de cobertura sobre las especies herbáceas.

En este sentido los modelos de crecimiento y rendimiento, que son de uso habitual en la actividad forestal, pueden representar la estructura de un rodal y su cambio a través 
del tiempo (Vanclay, 1994) y podrían ser útiles para predecir cambios en los niveles de cobertura. Por ejemplo, Waring y Schlesinger (1985) describen la relación específica entre el área de tejido conductivo y el área foliar. DeRose (2002), muestra como el índice de área foliar (IAF) esta relacionado con la densidad relativa, con valores crecientes del índice de área foliar a medida que aumenta la densidad.

Por otra parte Hale et al. (2009) relacionaron la transmisividad del dosel con parámetros sencillos de rodal, que eran especie- específicos. Para $P$. ponderosa, Laclau (2006) midió que aproximadamente un $70 \%$ de la biomasa aérea en árboles de entre 5 y $35 \mathrm{~cm}$ de dap se encontraba en el fuste, es decir que algunos parámetros que describen la estructura del rodal pueden emplearse para predecir los cambios en el sombreado a nivel del suelo. En este sentido la fotografía hemisférica permite estimar con precisión la cobertura de una forestación y mediante inventario forestal establecer cuáles son los parámetros de rodal característicos de dicha situación.

\section{OBJETIVOS}

El objetivo de este trabajo es diseñar un esquema de raleos a través de simulaciones, donde las intensidades y oportunidades de raleo están relacionados a los niveles de apertura del dosel de Pinus ponderosa, que son críticos para el crecimiento de Festuca pallescens.

\section{MATERIAL Y MÉTODO}

Para cumplir los objetovos trazados se avanzó en tres sentidos:

Establecer una relación matemática entre niveles de apertura del dosel y parámetros de rodal de $P$. ponderosa, que pueda ser incorporada al simulador "Piltriquitron" 1.0 (Andenmatten et al., 2007).

Calcular los umbrales críticos del crecimiento de F. pallescens en relación a la apertura del dosel de $P$. ponderosa.

Simular a partir de condiciones iniciales conocidas, el crecimiento del rodal, controlando que en ningún momento se superen los umbrales críticos para $F$. pallescens.

\section{Estimador del Nivel de Apertura del Dosel Mediante Parámetros de Rodal.}

Se dispuso de una base de datos de 109 sitios de muestreo del proyecto de INTA PNFOR 042151, de los cuales se seleccionaron 61 por su diversidad de tamaños, tratamientos de poda y raleo, calidades de sitio, y distribución geográfica (Figura Nro 1).

Los sitios se ubican en propiedades de productores rurales y empresas comerciales que colaboran con este proyecto. 
Las parcelas de inventario se establecieron dentro de los rodales, de tal forma que en el campo de visión de la foto hemisférica la estructura del rodal fuese homogénea. En el Cuadro Nro 1, se describen los parámetros de rodal.

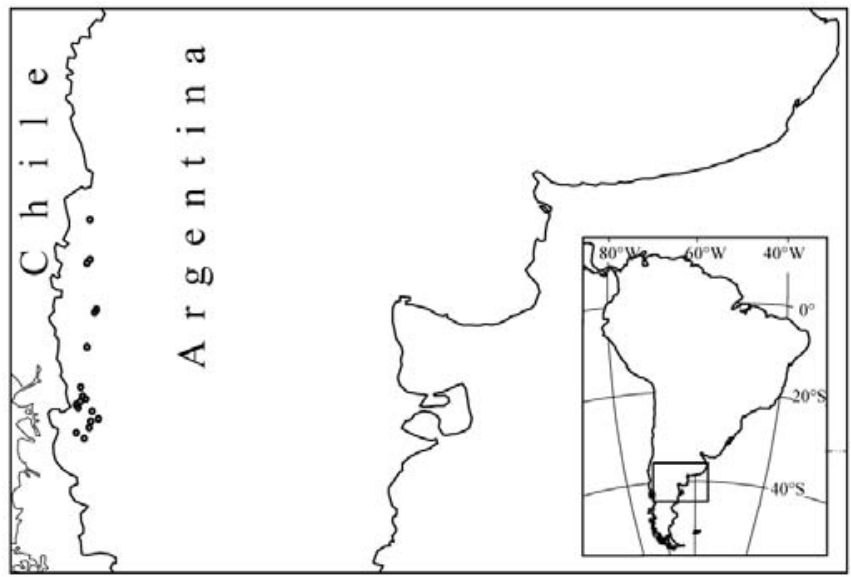

FIGURA $N^{\circ} 1$

MAPA DE UBICACIÓN DE RODALES

CUADRO $\mathrm{N}^{\circ} 1$

DESCRIPCIÓN DE BASE DE DATOS DE PARCELAS $(n=61)$

\begin{tabular}{|l|c|c|c|c|c|c|c|c|c|}
\cline { 2 - 10 } \multicolumn{1}{c|}{} & $\begin{array}{c}\mathbf{N} \\
{[\mathrm{arb} / \mathrm{ha}]}\end{array}$ & $\begin{array}{c}\mathrm{G} \\
{\left[\mathrm{m}^{2} \mathrm{ha}\right]}\end{array}$ & $\begin{array}{c}\mathrm{Dg} \\
{[\mathrm{cm}]}\end{array}$ & $\mathbf{D R}$ & $\begin{array}{c}\mathbf{V}\left[\mathrm{m}^{3} \mathrm{I}\right. \\
\mathrm{ha}]\end{array}$ & $\begin{array}{c}\text { ETOT } \\
{[\mathbf{a n ̃ o s}]}\end{array}$ & $\begin{array}{c}\mathbf{H 1 0 0} \\
{[\mathrm{m}]}\end{array}$ & $\begin{array}{c}\text { IS(20) } \\
{[\mathrm{m}]}\end{array}$ & $\begin{array}{c}\text { Apertura } \\
\text { del dosel } \\
{[\%]}\end{array}$ \\
\hline Media & 746 & 25 & 21 & 5,4 & 160 & 20 & 11,5 & 14,4 & 33,4 \\
\hline Mínimo & 259 & 4 & 7 & 1,2 & 20 & 9 & 4 & 6,6 & 14,8 \\
\hline Máximo & 2.581 & 98 & 40 & 21 & 720 & 37 & 28,4 & 21,9 & 79,1 \\
\hline Error típico & 55 & 2 & 1 & 0,5 & 20 & 1 & 0,7 & 3 & 2 \\
\hline
\end{tabular}

N: número de árboles/ha; G: área basal m²/ha; Dg: Diámetro del árbol de área basal promedio, cm.; DR: ïndice de Densidad Relativa de Curtis $\left(\mathrm{DR}=\mathrm{G} / \mathrm{Dg}^{-0,5}\right)$., Apertura de Dosel:, EAP: Edad a la altura del pecho, años. ETOT: Edad total, años. H100: altura dominante m., promedio de los 100 árboles más gruesos por hectárea. IS: Índice de sitio, es la $\mathrm{H} 100$ cuando el rodal tiene 20 años de edad al pecho, estimado utilizando la función de intercepción de crecimiento de acuerdo a Andenmatten y Letourneau (1997) y curvas de índice de sitio (Andenmatten y Letourneau, 1998).

Las fotos fueron tomadas con una Cámara digital Nikon Coolpix 5400, Lente hemisférico Delta T Device, UK. La cámara se ubicó en el centro de la parcela sobre un trípode con base nivelada. Las fotos fueron tomadas en condiciones de iluminación homogénea, apropiadas para la toma fotográfica. El procesamiento de las fotos se realizó con el programa Gap Light Anlyzer, desarrollado por Simon Fraser University, Institute 
of Ecosystem Studies. La selección del parámetro de rodal se basó en dos criterios, uno relacionado con los indicadores del ajuste estadístico. El otro criterio se basó en dos aspectos de la simulación, por un lado con la capacidad predictiva del simulador en relación al parámetro en cuestión. Por otro, con la capacidad operativa necesaria para incorporar este nuevo elemento en el simulador. Estos criterios fueron integrados a través de un puntaje que suma los valores asignados, dando mayor significación a los valores bajos, para seleccionar un parámetro.

En relación a los criterios estadísticos, se utilizó un orden por puntaje priorizando de forma conjunta la selección del parámetro de rodal que presentase el mayor $R^{2}$ ajustado y mayor precisión en la estimación de los datos para la validación. Además de los parámetros tradicionales de rodal (Cuadro $\mathrm{N}^{\circ} 1$ ), se incluyó la biomasa de hojas, el volumen de copa y la longitud de copa acumulada por hectárea, calculados a partir de ecuaciones de forma de copa y de biomasa por árbol (Letourneau et al, inédito).

Para todos los parámetros de rodal se empleó el mismo modelo, que fue del tipo exponencial:

$$
\text { Apertura del Dosel }[\%]=a \times X^{b}
$$

Donde:

a y b son coeficientes a ser ajustados.

X: es el parámetro de rodal a evaluar como estimador de la apertura del dosel.

Para el ajuste y validación se particionó la base de datos de forma aleatoria, se utilizaron 40 sitios para el procedimiento de ajuste, y los restantes 21 para estimar la precisión de la estimación.

La precisión se calculó de acuerdo a la ecuación Nro 2 como la proporción de observaciones del set de validación cuyo error de estimación estuviese comprendido entre $+-10 \%$ del valor observado.

Donde:

Pr ecisión de Estimación $[\%]=\left[\frac{\text { Cantidad de Errores Porcentuales }}{-10 \%}{ }_{-10 \%}^{+0 \%}\right] \times 100$

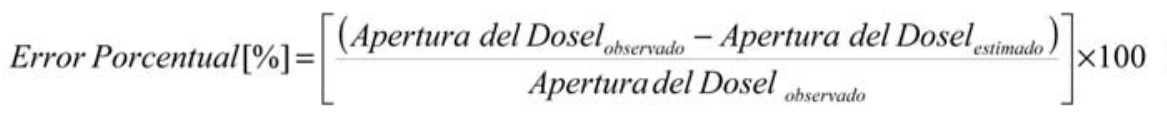

Donde: 
Apertura del Dosel [\%]: es el grado de apertura observado mediante fotografía hemisférica y estimada mediante la ecuación número 1.

Error porcentual: es la diferencia entre el valor observado y estimado expresado en porcentaje del valor observado.

Precisión de la estimación: es la proporción, respecto del total de datos del set validación, cuyos errores porcentuales se encuentran entre $\pm 10 \%$ del valor observado.

\section{Cálculo de los Umbrales Críticos para el Crecimiento de F. pallescens}

Para esto se dispuso de los datos de los estudios de Fernández (2003) y Fernández et al. (2006) y Caballé (inédito). Estos autores establecieron el pico de crecimiento relativo, valor integrado por el largo de hoja, el número de hojas por macollo y número de macollos por mata de F. pallescens.

Ambos estudios fueron realizados en el mismo sitio y puede considerarselos pertenecientes a una misma población, por lo que fueron agrupados, alcanzandose un total de 42 plantas medidas. Para cada estudio los valores de cobertura se reexpresaron en términos de apertura del dosel, y los crecimientos relativos se promediaron por nivel de apertura, con un total de siete valores promedios $(n=7)$ para el rango $0 \%-100 \%$ de apertura. Con estos datos consolidados se ajustó un modelo de crecimiento relativo para $F$. pallescens en función de niveles de apertura del dosel, este fue un polinomio de segundo grado con término constante:

$$
\text { crecimiento relativo }=c \times \text { Apertura Dosel }{ }^{2}+d \times \text { Apertura Dosel }+e
$$

Donde:

c, d y e, son coeficientes a ser ajustados

Apertura del dosel: como se definió con anterioridad.

\section{Simulación de Esquemas de Raleo}

Para predecir la evolución de la apertura del dosel se empleó el simulador Piltriquitron, que es un modelo de crecimiento y rendimiento de rodal basado en índice de sitio y la relación que existe entre la altura dominante e índices de la relación tamañoo - densidad de árboles, como el factor de espaciamiento y la densidad relativa de Curtis (1982), donde el crecimiento estimado a nivel de rodal se distribuye entre los individuos de acuerdo a su participación en la parcela. Para más referencias sobre los fundamentos del simulador puede consultarse Andenmatten et al. (2007).

Se seleccionaron cuatro situaciones de plantaciones de pino ponderosa a edades tempranas, con una densidad de plantas aproximada de $1.111 \mathrm{pl} / \mathrm{ha}$, con calidades de sitio distintas (Cuadro $N^{\circ} 2$ ). Estos datos se emplearon como condiciones iniciales, que se proyectaron hasta que la apertura del dosel llegaba a $30 \%$, Alcanzada esta condición se reprodujo la remoción de árboles de tal forma que aumente la apertura del dosel hasta 
niveles de $70 \%$. Esta operación se repitió hasta alcanzar un diámetro medio cuadrático de $45 \mathrm{~cm}$, que es el objetivo de producción establecido.

CUADRO N ${ }^{\circ} 2$

PARÁMETROS DE RODAL INICIALES PARA LA SIMULACIÓN

\begin{tabular}{|c|c|c|c|c|c|c|c|c|}
\hline Caso & $\begin{array}{c}\mathbf{N} \\
\text { [arb/ha] }\end{array}$ & $\begin{array}{c}\mathbf{G} \\
{[\mathrm{m} 2 / \mathrm{ha}]}\end{array}$ & $\begin{array}{c}\text { Dg } \\
\text { [cm] }\end{array}$ & DR & $\begin{array}{c}\text { ETOT } \\
\text { [años] }\end{array}$ & $\begin{array}{c}\text { H100 } \\
\text { [m] }\end{array}$ & $\begin{array}{c}\text { IS(20) } \\
\text { [m] }\end{array}$ & $\begin{array}{c}\text { Apertura de } \\
\text { Dosel [\%] }\end{array}$ \\
\hline 1 & 1.111 & 7,7 & 9,4 & 2,5 & 10 & 5,4 & 18,6 & 43 \\
\hline 2 & 1.001 & 3,6 & 6,8 & 1,4 & 9 & 4,0 & 16,4 & 64 \\
\hline 3 & 1.083 & 5,0 & 7,6 & 1,8 & 12 & 4,1 & 13,0 & 53 \\
\hline 4 & 1.111 & 7,6 & 9,3 & 2,5 & 12 & 4,0 & 13,0 & 43 \\
\hline
\end{tabular}

\section{RESULTADOS}

En relación a la selección del parámetro de rodal como predictor del nivel de apertura, la evaluación arrojó que el valor de $\mathrm{R}^{2}$ cuadrado ajustado más alto fue para el área basal $\left(R^{2}=0,752\right)$, luego la densidad relativa $\left(R^{2}=0,727\right)$ y el volumen/ha $\left(R^{2}=\right.$ $0,720)$. Le siguió el volumen de copa $\left(R^{2}=0,652\right)$, la biomasa de hojas $\left(R^{2}=0,596\right)$, el diámetro cuadrático medio $\left(R^{2}=0,558\right)$ y finalmente la longitud de copa $\left(R^{2}=0,534\right)$.

Sin embargo, para la precisión de la estimación del set de validación, el atributo de rodal más preciso fue el volumen de copa/ha con el $40 \%$ de las predicciones con errores de entre +- $10 \%$ del valor observado. Luego le siguió la longitud de copa por hectárea (35\%), la biomasa de hojas (30\%), la densidad relativa (25\%), el área basal (20\%), y finalmente el volumen por hectárea y el diámetro medio cuadrático (16 \% y 10 $\%$, respectivamente). Es decir que aquellos parámetros que cuantificaban la copa de los árboles fueron los más precisos.

En relación al cálculo de los parámetros por parte del simulador algunos de estos son proyectados y otros son deducidos matematicamente. Por ejemplo DR y V presentan la mejor calidad de predicción y facilidad de implementación en el formato actual del simulador, mientras que $\mathrm{G}$ y Dg son deducidos matematicamente. En orden de sencillez para la implemanteción siguen en orden decreciente el Vcopa, G, BHojas, y Dg. De acuerdo al puntaje para seleccionar el parámetro (Cuadro $N^{\circ} 3$ ) las mejores opciones son utilizar la densidad relativa DR y el volumen de copa Vcopa. Sin embargo se considera a los efectos de realizar este ejercicio de simulación, que el empleo de DR, brinda una mayor facilidad de implementación que supera a volumen de copa (Vcopa). 


\section{CUADRO $\mathrm{N}^{\circ} 3$ \\ PUNTAJES PARA SELECCIÓN DEL ATRIBUTO DE RODAL}

\begin{tabular}{|c|c|c|c|c|c|c|}
\hline $\mathbf{X}$ & $\mathbf{R 2}$ & $\begin{array}{c}\text { Precisión } \\
{[\% / \pm 10 \% \text { de }} \\
\text { error] }\end{array}$ & $\begin{array}{c}\text { Puntaje } \\
\text { R2 }\end{array}$ & $\begin{array}{c}\text { Puntaje } \\
\text { Precisión }\end{array}$ & $\begin{array}{c}\text { Puntaje } \\
\text { Implementación }\end{array}$ & $\begin{array}{c}\text { Puntaje } \\
\text { Total }\end{array}$ \\
\hline Dg [cm] & 0,558 & 11 & 6 & 7 & 2 & 15 \\
\hline Lcopa [m/ha] & 0,534 & 35 & 7 & 2 & 3 & 12 \\
\hline BHojas [Kg/ha] & 0,596 & 30 & 5 & 3 & 2 & 10 \\
\hline V [m3/ha] & 0,720 & 16 & 3 & 6 & 1 & 9 \\
\hline G [m2/ha] & 0,757 & 20 & 1 & 5 & 2 & 8 \\
\hline DR & 0,727 & 25 & 2 & 4 & 1 & 7 \\
\hline VCopa [m3/ha] & 0,652 & 42 & 4 & 1 & 2 & 7 \\
\hline
\end{tabular}

Lcopa, es la sumatoria de las longitudes de copa por hectárea,

BHojas, es la sumatoria de la biomasa de hojas por hectárea,

Vcopa: es la sumatoria de todos los volúmenes de copa por hectárea.

Los valores más bajos de puntaje tienen la mayor significancia para seleccionar el atributo de rodal.

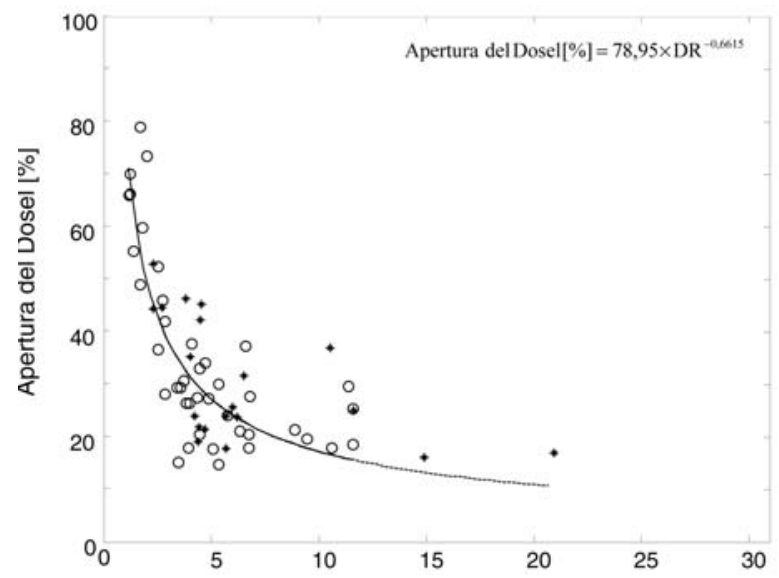

Símbolos vacíos datos del set de ajuste, puntos negros set de validación.

\section{FIGURA ${ }^{\circ} 3$}

\section{RELACIÓN ENTRE DENSIDAD RELATIVA Y APERTURA DEL RODAL.}

En relación a los umbrales críticos de $F$. pallescens (Figura $N^{\circ} 4$.) se oberva que las respuestas de crecimiento tienen una alta variancia, que no permiten establecer diferencias de crecimiento entre niveles de apertura, excepto para situaciones por debajo del $30 \%$ donde decrece fuertemente. A pesar de esto, en la Figura $\mathrm{N}^{\circ} 4$ se observa que los valores consolidados de crecimiento describen una tendencia. 
En condiciones de pastizal abierto el crecimiento promedio fue 2,3. Mientras que por debajo de una apertura de dosel de $30 \%$, las tasas de crecimiento relativo promedio son de 1,5, es decir un $35 \%$ menor que el pastizal sin cobertura. En condiciones intermedias entre $40 \%$ y $50 \%$ de apertura, la tasa de crecimiento relativo es de 2,9, siendo el más alto medido.

El modelo polinómico ajustado representa adecuadamente los datos disponibles e interpola en el rango entre $50 \%$ y $100 \%$, con el supuesto de la existencia de un máximo de crecimiento que se situaría al $70 \%$ de apertura del dosel con un crecimiento relativo estimado de 3,2.

En base a estos antecedentes se seleccionó como umbrales los valores de $30 \%$ de apertura como el mínimo para sostener el crecimiento de una mata de F. pallescens. El otro valor umbral se fijó en $70 \%$ de apertura, por ser un valor teórico al que podría alcanzarse las tasas de crecimiento más altas, se considera que por encima de $70 \%$ de apertura se pierde capacidad de producción de madera y no hay beneficio alguno sobre el pastizal. Estos valores umbrales se corresponden con valores de $\mathrm{DR}=1,20$ (70\% de apertura) y $\mathrm{DR}=4,32$ (30\% de apertura)

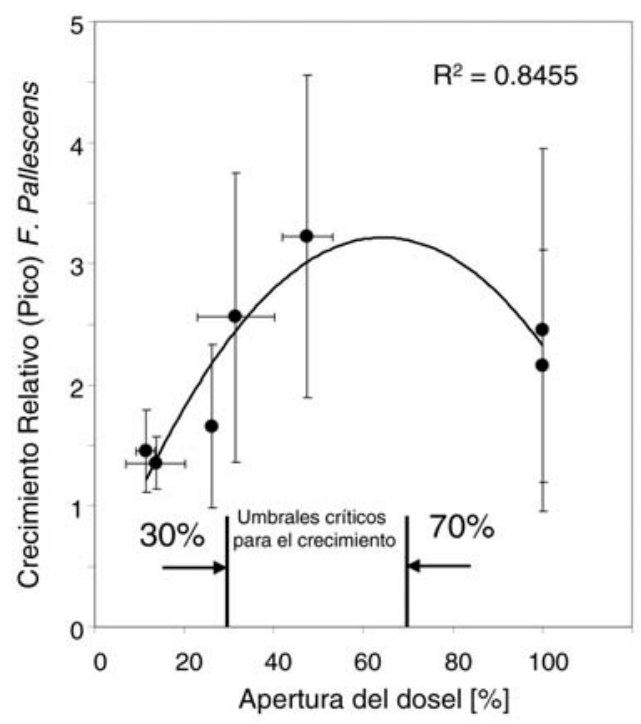

FIGURA N ${ }^{\circ} 4$

RELACIÓN ENTRE EL CRECIMIENTO RELATIVO DE MATAS DE F. pallescens Y LA APERTURA DEL DOSEL DE $P$. ponderosa.

La simulación de los 4 casos (Cuadro $\left.N^{\circ} 4\right)$ muestra que es necesario realizar al 
menos 2 raleos para sostener la apertura del dosel por encima de $30 \%$. Con un primer raleo entre los 13 y 18 años y un segundo raleo entre los 24 y 36 años, dependiendo este rango de la variación de calidad de sitio.

La producción bruta total se ubica entre 270 y $290 \mathrm{~m} 3 / \mathrm{ha}$, mientras que la producción comercial (hasta $15 \mathrm{~cm}$ punta fina) ronda entre los 175 a $200 \mathrm{~m}^{3} / \mathrm{ha}$, ambos incluyen el raleo.

Como simplificación de recomendación de raleo puede reexpresarse en función de la altura dominante del rodal, siendo independiente de la calidad de sitio.

El primer raleo se aplica cuando se alcanza los $8,2 \mathrm{~m}( \pm 0,7 \mathrm{~m})$ de altura dominante, mientras que el segundo raleo sucede a los $17,7 \mathrm{~m}( \pm 0,9 \mathrm{~m})$, alcanzandose la cosecha a los $21 \mathrm{~m}( \pm 0,6 \mathrm{~m})$, donde los datos son promedio (desvío estándar) de los cuatro casos simulados.

\section{CUADRO $\mathrm{N}^{\circ} 4$ \\ ESQUEMA DE RALEOS DE $P$. ponderosa \\ BASADO EN UMBRALES DE CRECIMIENTO DE F. pallescens}

\begin{tabular}{|c|c|c|c|c|c|}
\hline Caso & 1er raleo & 2 do raleo & Cosecha Final & $\begin{array}{c}\text { Producción. } \\
\text { Comercial [m3/ } \\
\text { ha] } \\
>15 \mathrm{~cm} \text { punta } \\
\text { fina, incluye } \\
\text { raleos }\end{array}$ & $\begin{array}{l}\text { Producción } \\
\text { Bruta [m3/ha], } \\
\text { incluye raleos }\end{array}$ \\
\hline 1 & $\begin{array}{l}13 \text { años } \\
1.111-290 \mathrm{pl} / \mathrm{ha} \\
\mathrm{d}=3,0 \mathrm{~m}-5,9 \mathrm{~m} \\
\mathrm{Dg}=13,9 \mathrm{~cm}\end{array}$ & $\begin{array}{l}24 \text { años } \\
290-77 \mathrm{pl} / \mathrm{ha} \\
\mathrm{d}=5,9 \mathrm{~m}-11,4 \mathrm{~m} \\
\mathrm{Dg}=34,0 \mathrm{~cm}\end{array}$ & $\begin{array}{l}29 \text { años } \\
77-0 \mathrm{pl} / \mathrm{ha} \\
\mathrm{Dg}=45,6 \mathrm{~cm}\end{array}$ & 196 & 290 \\
\hline 2 & $\begin{array}{l}15 \text { años } \\
1.001-248 \mathrm{pl} / \mathrm{ha} \\
\mathrm{d}=3,1 \mathrm{~m}-6,4 \mathrm{~m} \\
\mathrm{Dg}=15,3 \mathrm{~cm}\end{array}$ & $\begin{array}{l}29 \text { años } \\
248-68 \mathrm{pl} / \mathrm{ha} \\
\mathrm{d}=6,4 \mathrm{~m}-12,1 \mathrm{~m} \\
\mathrm{Dg}=36,8 \mathrm{~cm}\end{array}$ & $\begin{array}{l}33 \text { años } \\
68-0 \mathrm{pl} / \mathrm{ha} \\
\mathrm{Dg}=44,8 \mathrm{~cm}\end{array}$ & 195 & 287 \\
\hline 3 & $\begin{array}{l}18 \text { años } \\
1.083-290 \mathrm{pl} / \mathrm{ha} \\
\mathrm{d}=3,0 \mathrm{~m}-5,9 \mathrm{~m} \\
\mathrm{Dg}=13,9 \mathrm{~cm}\end{array}$ & $\begin{array}{l}36 \text { años } \\
290-78 \mathrm{pl} / \mathrm{ha} \\
\mathrm{d}=5,9 \mathrm{~m}-11,3 \mathrm{~m} \\
\mathrm{Dg}=33,6 \mathrm{~cm}\end{array}$ & $\begin{array}{l}45 \text { años } \\
78-0 \mathrm{pl} / \mathrm{ha} \\
\mathrm{Dg}=45,3 \mathrm{~cm}\end{array}$ & 185 & 276 \\
\hline 4 & $\begin{array}{l}17 \text { años } \\
1.111-280 \mathrm{pl} / \mathrm{ha} \\
\mathrm{d}=3,0 \mathrm{~m}-6,0 \mathrm{~m} \\
\mathrm{Dg}=14,3 \mathrm{~cm}\end{array}$ & $\begin{array}{l}35 \text { años } \\
280-75 \mathrm{pl} / \mathrm{ha} \\
\mathrm{d}=6,0 \mathrm{~m}-11,5 \mathrm{~m} \\
\mathrm{Dg}=34,5 \mathrm{~cm}\end{array}$ & $\begin{array}{l}43 \text { años } \\
75-0 \mathrm{pl} / \mathrm{ha} \\
\mathrm{Dg}=45,3 \mathrm{~cm}\end{array}$ & 175 & 267 \\
\hline
\end{tabular}

En cada celda de la tabla se describe la edad de raleo y cosecha, el número de plantas pre - post tratamiento $\mathrm{d}=$ distanciamiento promedio entre plantas,

Dg =: diámetro del arbol de área basal promedio, a esa edad. 


\section{DISCUSIÓN Y CONCLUSIONES}

\section{Selección del Atributo de Rodal y la Apertura del Dosel de $P$. ponderosa.}

La Densidad Relativa (DR) mostró ser una variable adecuada para realizar este ejercicio de simulación, sin embargo hay otros parámetros que también podrían servir para la estimación actual de la apertura de dosel de $P$. ponderosa.

Para el modelo empleado (Ecuación 1) el parámetro de rodal G área basal es el que más variación explica en la apertura del dosel, sin embargo en el simulador el área basal es un parámetro deducido matemáticamente y no forma parte de forma explícita en el algoritmo de proyección. El volumen de copa por hectárea (Vcopa) mostró ser el parámetro más preciso para estimar la apertura del dosel, este presenta una ventaja ante la DR y G, al ser capaz de representar el cambio de apertura de dosel en forma casi instantánea al aplicarse podas, y su empleo es preferible si se quiere integrar modelos más complejos como el de Brunner (1998) que permite estimar la intensidad de luz en condiciones de estructuras de árboles más heterogénas, como podría darse en plantaciones en cortinas u otros diseños.

\section{Umbrales Críticos para F. pallescens.}

Los datos de crecimiento relativo en relación a la apertura del dosel indican los rangos a considerar para sostener el crecimiento de F. pallescens. De la Figura $\mathrm{N}^{\circ} 4$ surge que habría un máximo de crecimiento que se produce a niveles de apertura del dosel de $70 \%$. Sin embargo si las condiciones climáticas son distintas a las aquí observadas, podría suceder que la interacción con $P$. ponderosa, cambie la intensidad y sentido (positivo - negativo). Callaway y Walker (1997) describen esta clase de cambios en la interacción entre plantas, cuando las condiciones de estrés abiótico se intensifican. En este caso podría utilizarse técnicas de sensibilización como las propuestas por Insúa et al (2008) para estudiar el efecto del cambio de interacción sobre el manejo del componente arbóreo.

\section{Simulación del Esquema de Raleo de $P$. ponderosa.}

Los resultados aquí obtenidos difieren en algunos aspectos con los de Laclau (1999) que son los antecedentes de análisis de sistemas sivopastoriles disponibles. Este realizó un análisis de viabilidad económica con el mismo simulador al aquí presentado. Unos de los esquemas que propone es una plantación de $1.111 \mathrm{pl} / \mathrm{ha}$, donde se extrae el $50 \%$ de las plantas cada vez, llegando a la corta final con 125 árboles, luego de tres raleos, pero establecía el turno de corta en función de la edad. En este trabajo se partió del supuesto que en una comunidad herbácea de Stipa speciosa var major, el umbral crítico inferior se sitúa al $25 \%$ de la Densidad Relativa máxima biológica, que de acuerdo a la ecuación aquí ajustada equivale a un valor de DR = 5 (27 \% de apertura), sin embargo para el umbral inferior lo situaron en $\mathrm{DR}=3,1$ que equivale $37 \%$ de apertura. Estos autores comentan que no disponían aún de información acerca del comportamiento de esta especie en estos rangos de apertura del dosel. 
En base a los resultados de sete trabajo es posible que el esquema de raleos propuesto por Laclau (1999) condicione en algún grado el crecimiento del pastizal, en el caso que este se tratara de F. pallescens.

Finalmente es necesario resaltar que existen vacíos de información como la respuesta del componente herbáceo a distintas condiciones climáticas, o la simulación del rodal que considere los efectos de la poda sobre el crecimiento. Por ello los resultados aquí presentados brindan información que debe ser tomada como orientadora para el manejo de los sistemas silvopastoriles compuestos por P. ponderosa y F. pallescens.

\section{AGRADECIMIENTOS:}

Los autores quieren agradecer a productores y empresas rurales del Noroeste de la Patagonia Andina Argentina que colaboraron en los estudios descriptos. Este proyecto se financió con fondos de proyectos nacionales de Sistemas Silvopastoriles y de Modelos de crecimiento y producción en plantaciones, pertenencientes al Programa Forestales del Instituto Nacional de Tecnología Agropecuaria, Argentina.

\section{REFERENCIAS}

Andenmatten, E., Letourneau, F.J., 1998. Curvas de índice de sitio y crecimiento en altura, para Pino Oregón (Pseudotsuga menziesii (Mirb) Franco), de aplicación en la región Andino Patagónica de Chubut y Río Negro, Argentina. Revista de Agronomía de la Universidad Nacional de La Plata, volumen 103(1): 69-75.

Andenmatten, E., Letourneau F.J., 1997. Funciones de intercepción del crecimiento, para la predicción de índice de sitio en pino ponderosa, de aplicación en la región Andino Patagónica de Río Negro y Chubut. Revista Quebracho, volumen 5, páginas 5-9.

Andenmatten, Ernesto; Letourneau, Federico; Getar, Edmundo, 2007. Simulador forestal para Pseudotsuga Menziesii (Mirb) Franco y Pinus ponderosa (Laws) en Patagonia Argentina. Resumen y trabajo completo En: Gonda, Héctor; Davel, Miguel; Loguercio, Gabriel; Picco, Omar A., (Eds) 1ra.Reunión sobre Forestación en la Patagonia. Ecoforestar 2007, ; Esquel, Chubut; CIEFAP; CFI; Universidad Nacional de la Patagonia San Juan Bosco; 25 al 27 de abril, p.374-380.

Brandle, J.R., Hodges, L., Zhou, 2001. Windbreaks in North American agricultural systems, Agroforestry Systems, Springer Netherlands, pp 65 - 78 Vol. 61-62.

Brunne, A., 1998. A light model for spatially explicit forest stand models, Forest Ecology and Management 107_1998.19-46

Curtis, R.O., 1982. Notes: A Simple Index of Stand Density for Douglas-fir, Forest Science, Volume 28, Number 1, 1 March 1982 , pp. 92-94(3).

DeRose, R., J., 2002. Leaf Area Index - Relative density relationships in evenaged Abies 
balsamea - Picea rubens stands in Maine. B.S. Utah State University, 2002. THESIS, The Graduate School The University of Maine December, 2004. pp 94

Fernández, M. E., 2003. Influencia del componente arbóreo sobre aspectos fisiológicos determinantes de la productividad herbácea en sistemas silvopastoriles de la Patagonia Argentina. Tesis Doctoral, Universidad Nacional del Comahue, Bariloche, Río Negro.

Fernández, M. E.; Gyenge, J. and Schlichter, T. M., 2006. Growth of Festuca pallescens in silvopastoral systems in Patagonia, Part 1: positive balance between competition and facilitation. Agroforestry Systems, 66, 259-269.

Hale, S.E, Edwards, C., Mason, W.L., Price, M., Peace, A., 2009. Relationships between canopy transmittance and stand parameters in Sitka spruce and Scots pine stands in Britain. Forestry 82(5): 503-513.

Holmgren, M., Scheffer, M., Huston, M.H., 1997. The interplay of facilitation and competition in plant communities. Ecology 78 (7), 1966-1975.

Insúa, D. R., Insúa, S.R. Jiménez, J.M, Martín, A.J., 2008. Simulación. Métodos y Aplicaciones. 2da Edición. RA-MA Pp 388.

Laclau, P., 1999. Capítulo II - Viabilidad Economica De Sistemas Silvopastoriles, en Informe Final Proyecto de Investigación aplicada (PIA) № 26/96, "Viabilidad ecológica y económica de la forestación con coníferas en sistemas silvopastoriles". Schlichter T.M., Laclau P. Dalla Salda G., Fernández M.E., Raffaele E., Gyenge J. Proyecto Forestal de Desarrollo / SAGPyA. Programa de Ámbito Nacional Forestal / INTA. Pp 31.

Laclau, P., 2006. Fijación en ecosistemas boscosos y herbáceos del norte de la Patagonia. Tésis doctoral. Universidad Nacional del Comahue Centro Regional Universitario Bariloche Depto. De postgrado - Doctorado en Biologia. Pp. 371.

Mosquera - Losada, M.R., Pinto - Tobalina, M. Rigueiro - Rodriguez, 2004. The herbaceous component in temperate silvopastoral systems pp 91-100. International Congress on Silvopastorim and Sustainable Management (2004: Lugo, Spain), Editado por M.R. Mosquera-Losada, A. Rigueiro-Rodriguez y J. McAdam.

Vanclay, J. K., 1994. "Modelling Forest Growth and Yield: Applications to Mixed Tropical, Forests," pp. 312. CAB International.

Valtorta, S. E., Leva, P.E., Gallardo, M.R., 1997. Evaluation of different shades to improve dairy cattle well-being in Argentina, International Journal of Biometeorology. Springer Berlin / Heidelberg, Earth and Environmental Science, pp 65-67. Vol 41(2).

Waring, R.H., Schlesinger, W.H., 1985. Forest Ecosystems. Concepts and Management. Academic Press, New York, p. 340. 
